\title{
Towards a marine synthesis of late Pliocene climate variability
}

\author{
Erin L. McClymont ${ }^{1}$, A.M. Haywood ${ }^{2}$ and A. Rosell-Melé ${ }^{3}$
}

PlioVAR meeting, Durham, UK, 19-21 April 2017

The aim of the PlioVAR working group is to develop a synthesis of our understanding of late Pliocene climate variability over glacial-interglacial timescales. Twenty-four researchers met to discuss progress in the synthesis of existing multi-proxy data sets from marine sediment archives, and to finalize agreement on common protocols for the reporting and compilation of data.

The Durham meeting started with presentations outlining PlioVAR aims and objectives, reporting progress towards regional data assimilation, and discussing appropriate data management. A white paper on potential stratigraphic protocols by Marci Robinson and Tim Herbert was outlined and discussed. An update on ongoing efforts by the inter-linked Pliocene Model Intercomparison Project (PlioMIP) was provided.

The remainder of the workshop focused on identifying the following three priorities:
- Continued data collection from marine sediment cores for late Pliocene climate (ca. 2.4-3.6 Ma)

During the northern summer, the regional synthesis leaders will continue to collate multi-proxy datasets. It was agreed that this information should not be restricted to seasurface temperatures, but span a range of climate variables where information is available. There was discussion around how to compare single-proxy and multi-proxy sites, how to handle potentially conflicting signals in multi-proxy sites, and how to represent uncertainties which are not always quantifiable. The two PlioVAR target intervals focus on (i) 2.4-2.8 Ma, to represent the intensification of northern hemisphere glaciation, and (ii) the marine isotope stages M2-KM3 interval (ca. 3.30-3.15 Ma), which marks a glacial-interglacial transition and had an orbital configuration similar to modern (Fig 1). Between 20 and 30 key marine sites have been identified which will contribute to the

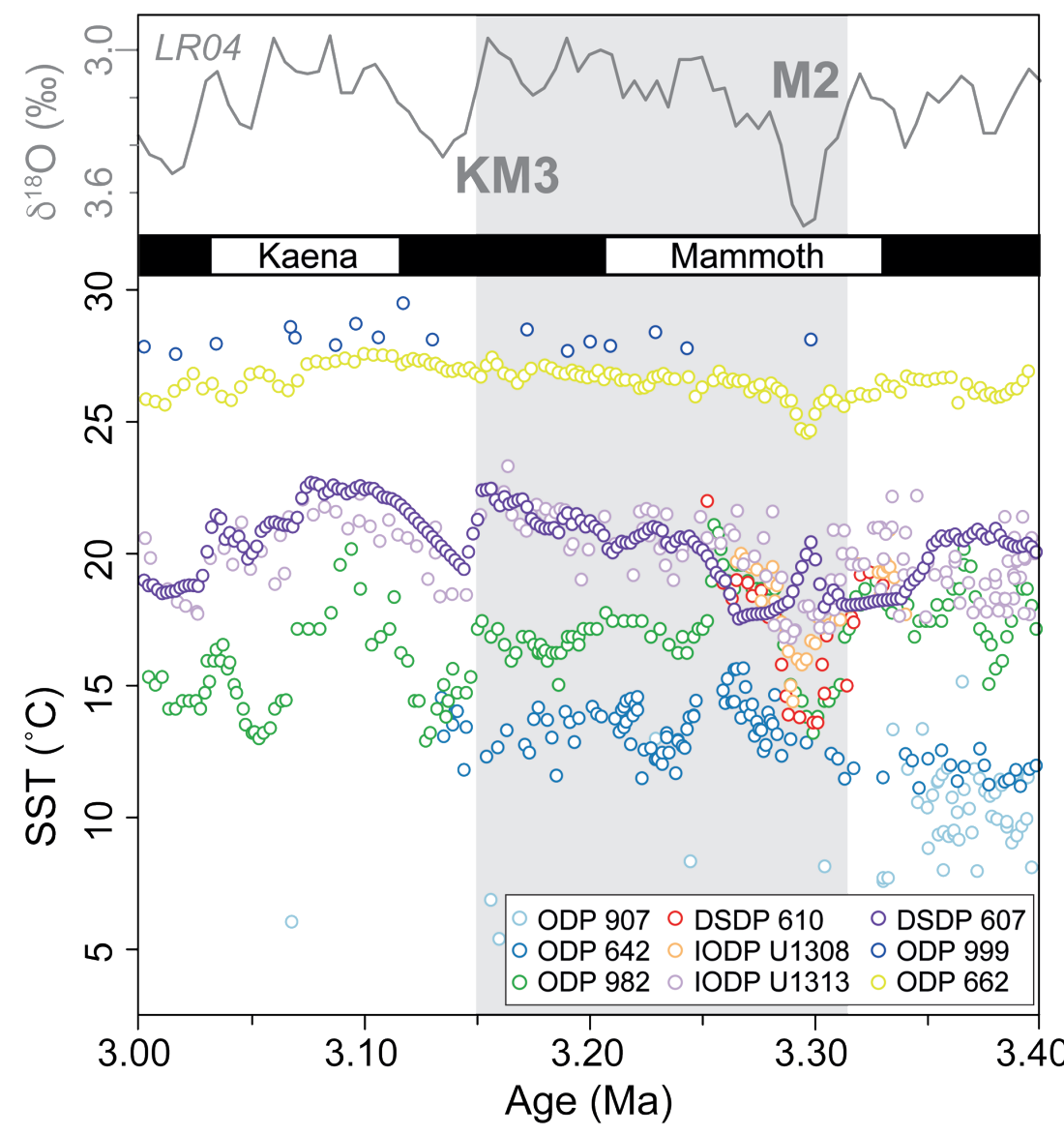

Figure 1: Ongoing data synthesis of late Pliocene climate variability, from the North Atlantic and Nordic Seas Top, LR04 global benthic stack with the targeted Marine Isotope Stage M2 to KM3 interval (grey shading) and paleomagnetic timescale. Bottom, compilation of sea surface temperature records between 3.40 and $3.00 \mathrm{Ma}$. PlioVAR will characterize the glacial-interglacial variability in these records across multiple proxies, and compare it to the intensification of northern hemisphere glaciation around 2.4-2.8 Ma. Source: De Schepper S, Ho SL and Risebrobakken B (unpublished).
PlioVAR synthesis. However, the heterogeneity of data availability across regions was highlighted. The highest number of sites are found in the North Atlantic (Fig. 1), whereas other regions have fewer sites represented. It was noted that recent International Ocean Discovery Program (IODP) expeditions will address some of these gaps (e.g. Expedition 353 Indian Monsoon, Expedition 354 Bengal Fan, Expedition 355 Arabian Sea Monsoon, Expedition 356 Indonesian Throughflow, Expedition 361 Southern African Climates).

\section{- Evaluation of stratigraphic controls}

A set of minimum standards was developed for the acceptance of datasets into the PlioVAR synthesis. Existing age models will be reviewed, and revised if required. It was recognized that there could be differences in the resolution of temporal control and the resolution of the proxy data, and the procedures for handling this were discussed.

\section{- Continued population of the PlioVAR database}

An interactive database which displays PlioVAR sites was shown, and will soon be launched. This resource will allow working group members to identify whether their own published or emerging datasets are missing from the current synthesis. For the data synthesis effort, a dataset template was agreed upon, which the leaders for each region will use, facilitating the statistical analysis required to investigate climate variability.

PlioVAR will continue to work on synthesis efforts over the next few months, and we welcome new participants to those activities. We are interested to learn of emerging data which might contribute to our planned assessment of glacial-interglacial variability within the two intervals detailed above. More information will be transmitted through the PAGES website and via the working group mailing list (www.pastglobalchanges.org/ini/ wg/pliovar/intro). The Steering Committee chair can also be contacted directly (erin. mcclymont@durham.ac.uk).

\section{ACKNOWLEDGEMENTS}

We thank PAGES, the Leverhulme Trust, and Durham University for the funding support which made this meeting possible.

\section{AFFILIATIONS}

'Department of Geography, Durham University, UK ${ }^{2}$ School of Earth \& Environment, University of Leeds, UK

${ }^{3}$ CREA and Universitat Autònoma de Barcelona, Spain CONTACT

Erin McClymont: erin.mcclymont@durham.ac.uk 